\title{
Treinamento auditivo em escolares com distúrbio de aprendizagem***
}

\author{
Auditory training in students with learning disabilities
}

\begin{abstract}
Fábio Henrique Pinheiro*
Simone Aparecida Capellini**
\end{abstract}

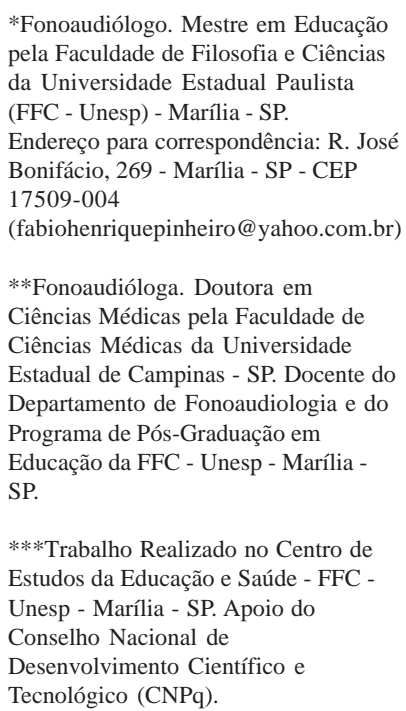

*Fonoaudiólogo. Mestre em Educação pela Faculdade de Filosofia e Ciências da Universidade Estadual Paulista (FFC - Unesp) - Marília - SP.

Endereço para correspondência: R. José Bonifácio, 269 - Marília - SP - CEP 17509-004

(fabiohenriquepinheiro@yahoo.com.br)

**Fonoaudióloga. Doutora em Ciências Médicas pela Faculdade de Ciências Médicas da Universidade Estadual de Campinas - SP. Docente do Departamento de Fonoaudiologia e do Programa de Pós-Graduação em Educação da FFC - Unesp - Marília SP.

***Trabalho Realizado no Centro de Estudos da Educação e Saúde - FFC Unesp - Marília - SP. Apoio do Conselho Nacional de Desenvolvimento Científico e Tecnológico (CNPq).

Artigo Original de Pesquisa

Artigo Submetido a Avaliação por Pares

Conflito de Interesse: não

Recebido em 13.02.2009.

Revisado em 25.08.2009; 03.12.2009; 19.01.2010.

Aceito para Publicação em 01.02.2010.

\section{Abstract}

Background: auditory training program in students with learning disabilities. Aim: to verify the efficacy of an auditory training program in students with learning disabilities; to compare the results of students with and without learning disabilities, who were and were not submitted to the auditory training program, in pre and post-testing. Method: participants were 40 students who were divided according to the following: GI, subdivided in: GIe (10 students with learning disabilities who were submitted to the program), GIc (10 students with learning difficulties who were not submitted to auditory training) and GII, subdivided in: GIIe (10 students without learning difficulties submitted to the auditory training program) and GIIc (10 students without learning difficulties who were not submitted to auditory training). The auditory training program Audio Training ${ }^{\circledR}$ was used. Results: the results indicate that GI presented a lower performance when compared to GII in activities related to auditory skills and phonological awareness. When comparing the pre and post-testing results, GIe and GIIe presented better performances in activities involving auditory skills and phonological awareness after the auditory training program. Conclusion: the performance of the students with learning disabilities in auditory and phonological tasks is lower when compared to the students without learning disabilities. The use of the auditory training program was effective and allowed students to develop these skills.

Key Words: Learning Disabilities; Intervention; Learning.

\section{Resumo}

Tema: programa de treinamento auditivo em escolares com distúrbio de aprendizagem. Objetivos: verificar a eficácia de um programa de treinamento auditivo em escolares com distúrbio de aprendizagem e comparar os achados dos procedimentos de avaliação utilizados nas pré e pós-testagem em escolares com distúrbio de aprendizagem e sem dificuldades de aprendizagem, submetidos e não submetidos ao programa de treinamento auditivo. Método: participaram deste estudo 40 escolares, sendo que esses foram divididos em: GI, subdividido em: GIe (10 escolares com distúrbio de aprendizagem submetidos ao programa de treinamento auditivo), GIc (10 escolares com distúrbio de aprendizagem não submetidos ao programa de treinamento auditivo) e GII, subdividido em: GIIe (10 escolares sem dificuldades de aprendizagem submetidos ao programa de treinamento auditivo) e GIIc (10 escolares sem dificuldades de aprendizagem não submetidos ao programa de treinamento auditivo). Foi realizado o programa de Treinamento Auditivo Audio Training ${ }^{\circledR}$. Resultados: os resultados mostraram que o GI apresentou desempenho inferior ao de GII em atividades relacionadas com as habilidades auditivas e de consciência fonológica. O GIe e o GIIe apresentaram melhor desempenho em habilidades auditivas e de consciência fonológica depois da aplicação do programa de treinamento auditivo, quando comparados os achados de pré e pós-testagem. Conclusão: o desempenho de escolares com distúrbio de aprendizagem nas tarefas auditivas e fonológicas apresenta-se inferior no que concerne ao de escolares sem distúrbio de aprendizagem. A utilização do programa de treinamento auditivo mostrou-se eficaz e possibilitou aos escolares o desenvolvimento dessas habilidades.

Palavras-Chave: Transtorno de Aprendizagem; Aprendizagem; Intervenção.

Referenciar este material como:

1 Pinheiro FH, Capellini SA. Treinamento auditivo em escolares com distúrbio de aprendizagem. Pró-Fono Revista de Atualização Científica. 2010 jan-mar;22(1):49$\sum 354$. 


\section{Introdução}

Na busca por minimizar as habilidades auditivas alteradas encontradas nos indivíduos com problemas de aprendizagem, um procedimento que tem sido proposto de maneira recorrente por diversos autores ${ }^{1-4}$ é o treinamento auditivo.

Os escolares com o diagnóstico de distúrbio de aprendizagem apresentam características alteradas em habilidades como a identificação ou decodificação da palavra, compreensão de leitura, cálculo, raciocínio matemático, soletração e expressão escrita, e ainda, podem ter acometidas áreas acadêmicas que envolvem, de maneira mais ampla, a expressão oral e a compreensão auditiva. A maneira que os indivíduos com distúrbio de aprendizagem processam e adquirem a informação é diferente do típico funcionamento esperado para uma criança ou adulto ${ }^{5-6}$.

Dessa forma, a associação entre dificuldades escolares e alteração no desenvolvimento das habilidades auditivas tem se tornado foco de trabalhos realizados com processamento auditivo ${ }^{7-8}$ e, mais recentemente, alguns estudos têm enfocado o trabalho com programas de treinamento auditivo ${ }^{1-4,9-}$ ${ }^{10}$. Esses estudos têm indicado a eficácia desses programas, mas ainda são escassas as pesquisas com esse enfoque na literatura nacional, principalmente quando se referem ao distúrbio de aprendizagem.

Com base no exposto acima, este estudo teve como objetivos verificar a eficácia de um programa de treinamento auditivo em escolares com distúrbio de aprendizagem e comparar os achados dos procedimentos de avaliação utilizados na pré e póstestagem em escolares com distúrbio de aprendizagem e sem dificuldades de aprendizagem, submetidos e não submetidos ao programa de treinamento auditivo.

\section{Método}

Este estudo foi aprovado pelo Comitê de Ética em Pesquisa da Faculdade de Filosofia e Ciências da Universidade Estadual Paulista (CEP - FFC Unesp) sob o protocolo número 2595/2007.

Participaram deste estudo 40 escolares na faixa etária de 8 a 14 anos de idade, de ambos os gêneros, de segunda a quarta séries do Ensino Fundamental de escolas municipais de Marilia - SP. Os escolares foram divididos em dois grupos: o Grupo I (GI), composto de 20 escolares com diagnóstico interdisciplinar de distúrbio de aprendizagem, de ambos os gêneros, que foram subdivididos em Grupo Ie (GIe), composto de 10 escolares com diagnóstico interdisciplinar de distúrbio de aprendizagem submetidos ao programa de treinamento auditivo; Grupo Ic (GIc), composto de 10 escolares com diagnóstico interdisciplinar de distúrbio de aprendizagem não submetidos ao programa de treinamento auditivo.

O diagnóstico de distúrbio de aprendizagem foi realizado por equipe interdisciplinar do Centro de Estudos da Educação e Saúde (Cees - Unesp) de Marília e do Ambulatório de Neurologia Infantil Desvios da Aprendizagem da Faculdade de Medicina (FM - Unesp) de Botucatu, incluindo avaliações fonoaudiológica, audiológica, neurológica e neuropsicológica. Os escolares foram caracterizados com distúrbio de aprendizagem quando, durante a avaliações, apresentaram resultados abaixo do esperado para a idade e escolaridade quanto à recepção e emissão de fala, leitura, escrita, cálculomatemático e memória de trabalho fonológica.

O Grupo II (GII), composto de 20 escolares sem dificuldades de aprendizagem, de ambos os gêneros, pareados segundo gênero e escolaridade com o GI e subdivididos em Grupo IIe (GIIe), composto de 10 escolares sem dificuldades de aprendizagem submetidos ao programa de treinamento auditivo; Grupo IIc (GIIc), composto de 10 escolares sem dificuldades de aprendizagem não submetidos ao programa de treinamento auditivo.

O grupo controle foi composto de escolares sem dificuldades de aprendizagem, que foram indicados pelas professoras de segunda a quarta série do ensino fundamental, com base no desempenho satisfatório em avaliações em dois bimestres consecutivos. Além disso, esses escolares não apresentavam histórico de deficiência auditiva, cognitiva, motora ou visual presentes em prontuário escolar.

A coleta de dados com os escolares foi realizada no Cees - Unesp, depois da assinatura do Termo de Consentimento Livre e Esclarecido pelos pais ou responsáveis pelos escolares.

Antes do início do estudo, os escolares do GI e GII foram submetidos ao exame audiológico básico: audiometria tonal liminar, logoaudiometria e medidas de imitância acústica, com resultados dentro do padrão de normalidade.

O procedimento utilizado para avaliação em situação de pré e pós-testagem foi a avaliação do processamento auditivo, composto de testes dicóticos verbais, sendo utilizado o teste dicótico de dígito e dissílabos alternados ${ }^{11}$ e a prova de consciência fonológica - instrumento de avaliação sequencial (Confias) ${ }^{12}$, com duração de quatro sessões de 50 minutos, sendo duas sessões para pré-testagem e duas sessões para pós-testagem.

Os grupos GIe e GIIe foram submetidos ao 
treinamento auditivo por meio do Software Audio Training ${ }^{\circledR}{ }^{13}$, em sala de terapia equipada com um computador e fones de ouvido para captação e recepção do estímulo acústico em todas as atividades. Esse programa foi utilizado por contemplar, de maneira ampla, as habilidades auditivas que podem estar acometidas na presença de alterações no processamento auditivo e também auxiliar a percepção auditiva da correspondência grafema-fonema, prérequisito importante para o ensino do sistema de escrita alfabético do Português ${ }^{14}$.

As atividades do programa foram realizadas individualmente em 18 sessões de 50 minutos. A frequência dos escolares do grupo submetido ao treinamento foi de duas vezes por semana. Asequência de atividade do treinamento auditivo foi igual à realizada para todos os escolares, conforme descrito a seguir:

. identificação do padrão de duração dos sons: o escolar foi instruído a indicar pela fala a categoria do som; e essa identificação ocorreu com a apresentação do som de maneira monaural (orelha direita seguida da orelha esquerda) e posteriormente de maneira binaural. Essa forma de exposição foi utilizada em todas as habilidades treinadas;

. identificação do padrão de frequência sonora: o escolar foi exposto por fone a 20 frequências (10 tons baixos e 10 tons altos) com a discriminação das frequências sonoras entre $880 \mathrm{~Hz}$ e $1122 \mathrm{~Hz}$. O escolar respondia por meio da nomeação dos estímulos (fino-grosso);

- evocação de sons não verbais em seqüência (memória para sons não verbais): foram apresentados ao escolar 20 pares com dois, três e quatro estímulos de sons não verbais. Concomitante à apresentação do som havia a apresentação de imagens, as quais o escolar deveria seqüencializar por meio da indicação das figuras correspondentes à seqüência sonora ouvida;

TABELA 1. Comparação intragrupo dos escolares de GIe, GIc, GIIe e GIIc no teste Dicótico de dígitos e SSW com o Teste de Mann-Whitney.

\begin{tabular}{c|c|c|c|c|c|c|c|c}
\hline \multirow{2}{*}{ Variável } & \multicolumn{7}{c}{ Par de Grupos } \\
\cline { 2 - 9 } & GIe x GIc & GIIe x GIIc & \multicolumn{2}{c}{ GIe X GIIe } & \multicolumn{2}{c}{ GIc x GIIc } \\
\cline { 2 - 9 } & DD & SSW & DD & SSW & DD & SSW & DD & SSW \\
\hline OD_PRE & 0,734 & 0,427 & 0,606 & 0,770 & $0,001^{*}$ & $0,001^{*}$ & $0,001^{*}$ & $0,001^{*}$ \\
OD_POS & $0,001^{*}$ & $0,001^{*}$ & $0,032^{*}$ & $0,018^{*}$ & $0,001^{*}$ & $0,001^{*}$ & $0,001^{*}$ & $0,001^{*}$ \\
OE_PRE & 0,448 & 0,363 & 0,395 & 0,376 & $0,003^{*}$ & $0,036^{*}$ & $0,001^{*}$ & $0,001^{*}$ \\
OE_POS & $0,002^{*}$ & $0,004^{*}$ & 0,398 & $0,004^{*}$ & $0,003^{*}$ & $0,001^{*}$ & $0,001^{*}$ & $0,001^{*}$ \\
CLPRE & 0,999 & 0,999 & $0,001^{*}$ & 0,999 & $0,001^{*}$ & $0,001^{*}$ & $0,001^{*}$ & $0,001^{*}$ \\
CLPOS & $0,029^{*}$ & $0,002^{*}$ & $0,004^{*}$ & 0,999 & $0,001^{*}$ & $0,030^{*}$ & $0,001^{*}$ & $0,001^{*}$ \\
\hline
\end{tabular}

Legenda: DD = dicótico de dígitos. SSW = dissílabos alternados. CL = Classificação
. discriminação e evocação de sons verbais: foi utilizada uma lista de palavras com os fonemas trabalhados conforme a ordem de desenvolvimento da fala e linguagem. Depois da apresentação das palavras, o escolar devia diferenciá-las, indicando se eram iguais ou diferentes, referindo posteriormente as palavras ouvidas;

- atenção auditiva: foram apresentadas aos escolares sequências de números e de meses do ano com a inserção de cortes, ou seja, o estímulo era apresentado sequencialmente, porém com partes omitidas selecionadas previamente. O escolar devia indicar os momentos de corte e evocar os números e os meses que haviam sido suprimidos. Foi utilizado também um texto ao qual o escolar deveria permanecer atento, pois sua compreensão seria avaliada posteriormente por meio de perguntas relacionadas com o seu conteúdo.

Os resultados foram analisados estatisticamente pelo programa SPSS (Statistical Package for Social Sciences), em sua versão 13.0. Também foi utilizado o Teste de Mann-Whitney e teste dos postos sinalizados de Wilcoxon. Foi adotado o nível de significância de 5\% $(0,050)$ para a aplicação dos testes estatísticos(*).

\section{Resultados}

Os resultados evidenciaram melhora do desempenho dos escolares submetidos ao treinamento ao compararmos (GIe e GIIe) em situação de pré e pós testagem, e na comparação com seus respectivos grupos controle (GIc e GIIc) nos testes dicóticos de dígitos e dissílabos alternados, conforme observado na Tabela 1.

Na Tabela 2, os resultados demonstraram diferença estatisticamente significante de GIe no que concerne ao GIc nos subtestes de produção de rima e transposição de sílabas, evidenciando um desempenho superior de GIe. Ao analisar o escore total dos subtestes realizados, verificou-se que os grupos GIe e GIIe apresentaram médias de desempenho superiores relativas ao GIc e GIIc depois da aplicação do programa de treinamento auditivo.

Com relação à média de tempo para execução das tarefas, verificou-se que o grupo GII apresenta uma média de tempo utilizada inferior à do grupo GI, ratificando o melhor desempenho de GII nas provas realizadas.

Com relação ao desempenho nos subtestes fonêmicos da prova de consciência fonológica instrumento de avaliação sequencial (Confias), verificou-se diferença estatisticamente significante 
nos resultados de GIe em relação ao GIc nos subtestes de síntese fonêmica, transposição fonêmica e no escore total dos subtestes de habilidades fonêmicas.

Ao realizar a comparação dos desempenhos dos grupos GIIe e GIIc, verificou-se diferença estatisticamente significativa nos subtestes de identificação de fonema final, segmentação fonêmica e transposição fonêmica, sugerindo melhor desempenho de GIIe. O escore total dos subtestes de habilidades fonêmicas apresentados pelos grupos GIIe e GIe depois do treinamento auditivo evidenciou diferença estatisticamente significante em situação de pós-testagem, indicando melhor desempenho dos grupos submetidos ao treinamento auditivo.

Com relação à média de tempo para execução das tarefas, foi verificado que o grupo GII realizou as atividades em uma média de tempo inferior à do GI, ratificando, assim, o melhor desempenho de GII nas provas realizadas, como pode ser verificado na Tabela 3.

TABELA 2. Distribuição das médias, desvios-padrão e significância (p) do desempenho na pós-testagem dos grupos GIe, GIc, GIIe e GIIc nos subtestes silábicos do Confias.

\begin{tabular}{|c|c|c|c|c|c|}
\hline & Habilidade & Grupos & Média & Desvio-Padrão & Valor de $\mathrm{p}$ \\
\hline \multirow{44}{*}{ habilidades silábicas } & \multirow[t]{4}{*}{ síntese silábica } & GIe & 4,00 & 0,00 & \multirow[t]{2}{*}{0,317} \\
\hline & & GIc & 3,80 & 0,63 & \\
\hline & & GIIe & 4,00 & 0,00 & \multirow[t]{2}{*}{$>0,999$} \\
\hline & & GIIc & 4,00 & 0,00 & \\
\hline & \multirow[t]{4}{*}{ segmentação silábica } & GIe & 4,00 & 0,00 & \multirow[t]{2}{*}{0,317} \\
\hline & & GIC & 3,70 & 0,95 & \\
\hline & & GIIe & 4,00 & 0,00 & \multirow[t]{2}{*}{$>0,999$} \\
\hline & & GIIC & 4,00 & 0,00 & \\
\hline & \multirow[t]{4}{*}{ identificação de sílaba inicial } & GIe & 3,70 & 0,48 & \multirow[t]{2}{*}{0,259} \\
\hline & & GIc & 3,20 & 1,03 & \\
\hline & & GIIe & 4,00 & 0,00 & \multirow[t]{2}{*}{0,147} \\
\hline & & GIIC & 3,70 & 0,67 & \\
\hline & \multirow[t]{4}{*}{ identificação de rima } & GIe & 3,10 & 0,88 & \multirow[t]{2}{*}{0,745} \\
\hline & & GIc & 2,90 & 1,20 & \\
\hline & & GIIe & 4,00 & 0,00 & \multirow[t]{2}{*}{0,068} \\
\hline & & GIIc & 3,60 & 0,70 & \\
\hline & \multirow[t]{4}{*}{ produção de palavra com a sílaba fornecida } & GIe & 3,70 & 0,95 & \multirow[t]{2}{*}{0,358} \\
\hline & & GIc & 3,50 & 0,85 & \\
\hline & & GIIe & 4,00 & 0,00 & \multirow[t]{2}{*}{$>0,999$} \\
\hline & & GIIc & 4,00 & 0,00 & \\
\hline & \multirow[t]{4}{*}{ identificação de sílaba medial } & GIe & 2,30 & 0,95 & \multirow[t]{2}{*}{0,164} \\
\hline & & GIc & 1,60 & 1,17 & \\
\hline & & GIIe & 3,60 & 0,52 & \multirow[t]{2}{*}{0,131} \\
\hline & & GIIc & 3,90 & 0,32 & \\
\hline & \multirow[t]{4}{*}{ produção de rima } & GIe & 2,30 & 0,48 & \multirow[t]{2}{*}{$0,002^{*}$} \\
\hline & & GIc & 0,70 & 1,06 & \\
\hline & & GIIe & 3,90 & 1,60 & \multirow[t]{2}{*}{0,212} \\
\hline & & GIIc & 3,00 & 1,05 & \\
\hline & \multirow[t]{4}{*}{ exclusão de sílaba } & GIe & 4,70 & 2,06 & \multirow[t]{2}{*}{0,485} \\
\hline & & GIc & 4,10 & 2,38 & \\
\hline & & GIIe & 7,00 & 1,25 & 0,129 \\
\hline & & GIIc & 6,10 & 1,60 & \\
\hline & transposição de sílabas & GIe & 2,60 & 1,17 & $0,040 *$ \\
\hline & & GIc & 1,30 & 1,25 & \\
\hline & & GIIe & 3,70 & 0,48 & $>0,999$ \\
\hline & & GIIc & 3,70 & 0,48 & \\
\hline & escore total do nível silábica & GIe & 30,10 & 5,13 & 0,087 \\
\hline & & GIc & 24,60 & 8,28 & \\
\hline & & GIIe & 38,20 & 1,40 & 0,370 \\
\hline & & GIIc & 37,00 & 2,54 & \\
\hline & tempo & GIe & 24,60 & 4,25 & 0,519 \\
\hline & & GIc & 26,60 & 7,18 & \\
\hline & & GIIe & 15,00 & 2,00 & 0,122 \\
\hline & & GIIC & 17,50 & 4,99 & \\
\hline
\end{tabular}


TABELA 3. Distribuição das médias, desvios-padrão e significância (p) do desempenho na pós-testagem dos grupos GIe, GIc, GIIe e GIIc nos subtestes fonêmicos do Confias.

\begin{tabular}{|c|c|c|c|c|c|}
\hline & Habilidade & Grupos & Média & Desvio-Padrão & Valor de $\mathrm{p}$ \\
\hline \multirow{36}{*}{ habilidades fonêmicas } & \multirow[t]{4}{*}{ produção da palavra com som fornecido } & GIe & 3,30 & 0,82 & \multirow[t]{2}{*}{0,336} \\
\hline & & GIc & 2,70 & 1,34 & \\
\hline & & GIIe & 4,00 & 0,00 & \multirow[t]{2}{*}{$>0,999$} \\
\hline & & GIIc & 4,00 & 0,00 & \\
\hline & \multirow[t]{4}{*}{ identificação de fonema inicial } & GIe & 3,00 & 0,94 & \multirow[t]{2}{*}{0,158} \\
\hline & & GIC & 2,30 & 1,16 & \\
\hline & & GIIe & 4,00 & 0,00 & \multirow[t]{2}{*}{0,146} \\
\hline & & GIIc & 3,80 & 0,42 & \\
\hline & \multirow[t]{4}{*}{ identificação de fonema final } & GIe & 2,30 & 0,48 & \multirow[t]{2}{*}{0,064} \\
\hline & & GIc & 1,70 & 0,82 & \\
\hline & & GIIe & 3,40 & 0,52 & \multirow[t]{2}{*}{$0,039^{*}$} \\
\hline & & GIIc & 2,70 & 0,82 & \\
\hline & \multirow[t]{4}{*}{ exclusão de fonema } & GIe & 2,90 & 1,60 & \multirow[t]{2}{*}{0,067} \\
\hline & & GIc & 1,60 & 1,51 & \\
\hline & & GIIe & 5,00 & 0,94 & \multirow[t]{2}{*}{$>0,999$} \\
\hline & & GIIc & 5,00 & 0,82 & \\
\hline & \multirow[t]{4}{*}{ síntese fonêmica } & GIe & 2,10 & 0,57 & \multirow[t]{2}{*}{$0,012^{*}$} \\
\hline & & GIc & 1,20 & 0,79 & \\
\hline & & GIIe & 2,70 & 0,48 & \multirow[t]{2}{*}{0,067} \\
\hline & & GIIc & 2,20 & 0,63 & \\
\hline & \multirow[t]{4}{*}{ segmentação fonêmica } & GIe & 0,00 & 0,00 & \multirow[t]{2}{*}{$>0,999$} \\
\hline & & GIc & 0,00 & 0,00 & \\
\hline & & GIIe & 1,70 & 0,95 & \multirow[t]{2}{*}{$0,036 *$} \\
\hline & & GIIC & 0,80 & 0,79 & \\
\hline & \multirow[t]{4}{*}{ transposição fonêmica } & GIe & 1,00 & 0,82 & \multirow[t]{2}{*}{$0,002^{*}$} \\
\hline & & GIc & 0,00 & 0,00 & \\
\hline & & GIIe & 1,20 & 1,03 & \multirow[t]{2}{*}{$0,002^{*}$} \\
\hline & & GIIc & 0,00 & 0,00 & \\
\hline & \multirow[t]{4}{*}{ escore total do nível do fonema } & GIe & 16,20 & 5,33 & \multirow[t]{2}{*}{$0,004^{*}$} \\
\hline & & GIc & 9,40 & 3,86 & \\
\hline & & GIIe & 22,00 & 2,49 & \multirow[t]{2}{*}{$0,004 *$} \\
\hline & & GIIc & 18,80 & 1,62 & \\
\hline & \multirow[t]{4}{*}{ tempo } & GIe & 24,60 & 4,25 & \multirow[t]{2}{*}{0,519} \\
\hline & & GIC & 26,60 & 7,18 & \\
\hline & & GIIe & 15,00 & 2,00 & \multirow[t]{2}{*}{0,122} \\
\hline & & GIIc & 17,50 & 4,99 & \\
\hline
\end{tabular}

\section{Discussão}

Os achados deste estudo revelaram que os escolares com diagnóstico de distúrbio de aprendizagem apresentaram um atraso no desenvolvimento das habilidades auditivas, o que pode impedir o processamento adequado das informações e, em consequência, pode afetar o desenvolvimento normal da linguagem e da escrita, visto que para que o escolar aprenda adequadamente na escola ele deve apresentar um bom desenvolvimento de suas habilidades auditivas ${ }^{13,15}$.

De acordo com a literatura internacional ${ }^{5-4,16}$, a média de desempenho nas provas de processamento auditivo dos escolares com distúrbio de aprendizagem é inferior à de escolares normais. Esse fato pode ser verificado nos achados deste estudo quando comparados os desempenhos dos grupos com e sem distúrbio de aprendizagem, sendo verificada a prevalência de um desempenho superior por parte dos escolares sem dificuldades.
A análise dos resultados das provas auditivas dos escolares do grupo com diagnóstico de distúrbio de aprendizagem sugere a ineficácia da integração de informações auditivas por meio do desempenho desses grupos nos testes de Dicóticos de Dígitos e Dissílabos Alternados. No entanto, em condições de pós-testagem, as médias de desempenho nos testes de processamento auditivo dos escolares com distúrbio de aprendizagem melhoraram no que se refere à pré-testagem.

Inúmeros estudos ${ }^{1-4,9}$ têm trazido dados semelhantes sobre a eficácia da utilização de programas de treinamento auditivo e seus efeitos nos testes de avaliação do processamento auditivo, corroborando os achados deste estudo.

Alguns escolares com problemas de leitura apresentam menor extensão de memória para material apresentado auditivamente e tendem a ter um desempenho inferior em tarefas que requeiram consciência fonológica ${ }^{17}$. Os escolares que 
apresentam dificuldades em processar os estímulos sonoros da fala poderão deparar-se com obstáculos na segmentação e manipulação da estrutura fonológica da linguagem e, conseqüentemente, estarão sujeitas a apresentar dificuldades de leitura e escrita ${ }^{18-19}$. Isso pode ser verificado nos resultados obtidos com a aplicação do CONFIAS.

O desempenho dos escolares nas tarefas de consciência fonológica mostrou que as performances dos escolares de todos os grupos foram superiores nas tarefas silábicas no que concerne às tarefas fonêmicas, 0 que está de acordo com os achados na literatura ${ }^{12,20}$.

Ao comparar a média do total de desempenhos nos subtestes silábicos, verificou-se desempenho superior dos grupos experimentais com relação aos seus respectivos grupos controle, indicando melhora na média de desempenho nos grupos que foram submetidos ao programa de treinamento auditivo, sendo o mesmo achado encontrado com relação ao desempenho nas habilidades fonêmicas.

\section{Referências Bibliográficas}

1. Hayes EA, Warrier CM, Nicol TG, Zecker SG, Kraus N. Neural plasticity following auditory training in children with learning problems. Clin Neurophs. 2003; (114): 673-48.

2. Moore DR, Amitay S. Auditory training: rules and applications. Seminar in Hearing. 2007; (28) 2: 99-109.

3. Zalcman TE. \& Schochat E. A eficácia do treinamento auditivo formal em indivíduos com transtorno de processamento auditivo, Revista da Sociedade Brasileira de Fonoaudiologia. 2007; 12 (4): 310-14.

4. Moore DR, Ferguson MA, Halliday LF, Rilley A. Frequency discrimination in children: Perception, learning and attention. Hearing Research. 2008; (238): 147-54.

5. Silver CH, Ruff RM, Iveson GL, Barth JT, Broshek, DK, Bush SS, Koffer SP, Reynolds, CR. Learning disabilities: The need for neuropsycological evaluation. Archives of Clinical Neuropsychology. 2008; (23): 217-19.

6. Wu TK, Huang SC, Meng YR.. Evaluation of ANN and SVM classifiers as predictors to the diagnosis of studentes with learning disabilities. Expert Systems with Appplications. 2008; (34): 846-56.

7. Ramos CS, Pereira LD. Processamento auditivo e audiometria de altas freqüências em escolares de São Paulo. Pró-Fono Revista de Atualização Científica. 2005 maioago; 17 (2): 153-64.

8. Sauer L, Pereira LD, Ciasca SM, Pestun M, Guerreiro MM. Processamento auditivo e SPECT e crianças com dislexia. Arquivos de. Neuropsiquatria. 2006; 64 (1):108-111.

9. Agnew JA, Dorn C, Eden GF. Effect of intensive training on auditory processing and reading skills. Brain Lang. 2004; (88): 21-25.

10. Berlau KM, Weinberger NM. Learning strategy determines auditory cortical plasticity. Neurobiology of learning and memory. 2008; (89): 153-166.
Esses resultados sugerem um desempenho mais adequado desses escolares com relação aos níveis de atenção auditiva, percepção dos sons da fala, reconhecimento de palavras e compreensão auditiva.

\section{Conclusão}

Os achados deste estudo permitem concluir que: os escolares com distúrbio de aprendizagem e sem distúrbio submetidos ao programa de treinamento auditivo apresentaram melhora de seu desempenho em situação de pós-testagem em comparação com os escolares que não foram submetidos ao programa de ambos os grupos, evidenciando, assim a eficácia do programa de treinamento auditivo com o software utilizado; os escolares com distúrbio de aprendizagem apresentaram desempenho inferior se comparados com os escolares sem dificuldades de aprendizagem em provas de consciência fonológica e processamento auditivo.
11. Pereira LD, Schochat E. Processamento Auditivo Central: manual de avaliação. São Paulo: Lovise, 1997.

12. Moojen S, Lamprecht R, Santos RM, Freitas GM, Brodacz R, Siqueira M, et al. Consciência fonológica: Instrumento de avaliação seqüencial. São Paulo: Casa do Psicólogo, 2003.

13. Nunes C, Frota S. Audio Training: fundamentação teórica e prática. São Paulo: AM3 Artes, 2006

14. Capellini SA. Distúrbios de aprendizagem versus dislexia. In: Ferreira LP, Befi-Lopes D, Limongi S, organizadores. Tratado de fonoaudiologia. São Paulo: Ed. Roca; 2004. p. 862-76.

15. Santos MFC, Pereira LD. Escuta com dígitos. In: Pereira LD, Schochat E. Processamento auditivo central: manual de avaliação. São Paulo: Ed. Lovise; 1997. p.147-50.

16. Dawes P, Bishop DVM, Sirimanna T, Bamiou DE. Profile and aetiology of children diagnosed with auditiry processing disorder (APD). Int J Pediatr Otorhinolaryngol. 2008; (72): 483-489.

17. Nunes MVRS. A aprendizagem de leitura e o "loop" fonológico. RFML, série III. 2001; 6 (1): 21-28.

18. Cherry R, Rubinstein A. Comparing Monotic and Diotic Selective Auditory Attention Abilities in Children. Lang. Speeech and Hear. Serv. Sch. 2006; (37): 137-42.

19. Idiazábal-Aletxa MA, Saperas-Rodrígues, M. Procesamiento auditivo em el trastorno específico del lenguaje. Revista de Neurología. 2008; 46 (supl.1): 91-5.

20. Tirapegui CJC, Fajardo LRC, Ortiz ZDB. Conciencia fonológica y lengua en niños con trastorno específico del lenguaje expressivo. Rev. Cefac. 2005 out-dez; 7(4): 419-25. 